\section{References}

1 Okoli K, Gupta A, Irani F, Kasmani R. Immune thrombocytopenia associated with Mycoplasma pneumoniae: a case report and review of literature. Blood Coagul Fibrinolysis 2009;20:595-8.

2 Izumikawa K, Izumikawa K, Takazono $\mathrm{T}$ et al. Clinical features, risk factors and treatment of fulminant Mycoplasma pneumoniae pneumonia: A review of Japanese literature. J Infect Chemother 2014;20:181-5.

3 Kenney RT, Li JS, Clyde WA Jr et al. Mycoplasmal pericarditis: evidence of invasive disease. Clin Infect Dis 1993;17:S58-62.
4 Hawkins S, Rausch CM, McCanta AC. Constrictive pericarditis secondary to infection with Mycoplasma pneumoniae. Curr Opin Pediatr 2011;23:126-9.

Address for correspondence: Dr V Sawhney, Cardiology, St Bartholomew's Hospital, 1st Floor, 60 Dominion House West, Smithfields, London EC1A 9BE.

Email: sawhneyvin@googlemail.com

\title{
Lesson of the month 2: Severe reactivation of hepatitis B after immunosuppressive chemotherapy
}

\author{
Authors: Jessica Katharine Dyson, ${ }^{A}$ Mark Hudson ${ }^{B}$ and Stuart McPherson ${ }^{C}$
}

\section{Patients with current or past hepatitis B virus (HBV) infection are at risk of viral reactivation if they receive immune-modulating treatment or chemotherapy. This can range from subclinical elevation in HBV DNA levels, to abnormal liver function tests, to severe hepatitis with liver failure and risk of death. All patients should be screened for hepatitis B with surface antigen and core antibody before receiving immunosuppression. Patients with positive hepatitis $B$ serology should be referred for specialist advice. Prophylactic antiviral treatment is recommended for patients with current/past hepatitis $B$ who receive immunosuppressive chemotherapy.}

KEYWORDS: Hepatitis B, reactivation, immunosuppression, chemotherapy, antiviral therapy.

\section{Introduction}

Patients with current or past hepatitis B virus (HBV) infection are at risk of viral reactivation if they receive immune-modulating treatment or chemotherapy. This can range from subclinical elevation in HBV DNA levels to abnormal liver function tests (LFTs), to severe hepatitis with liver failure and risk of death.

\footnotetext{
Authors: ${ }^{\text {A }}$ clinical hepatology fellow, Freeman Hospital, Newcastle upon Tyne Hospitals NHS Foundation Trust, Newcastle upon Tyne, UK; ${ }^{B}$ consultant hepatologist, Freeman Hospital, Newcastle upon Tyne Hospitals NHS Foundation Trust and Institute of Cellular Medicine, Newcastle University, Newcastle upon Tyne, UK; ${ }^{C}$ consultant hepatologist, Freeman Hospital, Newcastle upon Tyne Hospitals NHS Foundation Trust and Institute of Cellular Medicine, Newcastle University, Newcastle upon Tyne, UK
}

\section{Case report}

A 72-year-old man was noted to have submandibular gland swelling at a dental appointment. Further assessment showed widespread lymphadenopathy and excision biopsy diagnosed diffuse large B-cell lymphoma. His past medical history included pulmonary embolus.

He was planned for six cycles of R-CHOP chemotherapy (rituximab, cyclophosphamide, doxorubicin [hydroxydaunomycin], vincristine, prednisolone). Before starting treatment, hepatitis B serology was checked; he was anti-HBcAb (hepatitis B core antibody) positive, HBsAg (hepatitis B surface antigen) negative, anti-HBsAb (hepatitis B surface antibody) negative with undetectable HBV DNA $(<20 \mathrm{IU} / \mathrm{ml})$, in keeping with past HBV infection. LFTs were normal. The virology report stated: 'consistent with past HBV infection, but risk of reactivation on immunosuppression. Please ensure HBsAg and LFTs are monitored.'

After four cycles of R-CHOP (January 2013), tests for HBsAg were positive and HBV DNA was $1.2^{\prime} 10^{7} \mathrm{IU} / \mathrm{ml}$, consistent with acute $\mathrm{HBV}$ reactivation. The patient was started on tenofovir $245 \mathrm{mg}$ daily.

He completed six cycles in March 2013 and positron emission tomography computed tomography (PET-CT) confirmed good radiological response. However, in May 2013 he was admitted with jaundice and international normalised ratio (INR) $>10$. Blood tests showed significant liver dysfunction (bilirubin $105 \mu \mathrm{mol} / \mathrm{l}$, alanine aminotransferase [ALT] $988 \mathrm{U} / \mathrm{l}$, alkaline phosphatase [ALP] $317 \mathrm{U} / \mathrm{l}$ and albumin $35 \mathrm{~g} / \mathrm{l})$. HBV DNA had fallen to 5,300 IU/ml. An abdominal CT scan showed no biliary obstruction or other cause for jaundice. Tests for other hepatotrophic viruses were negative. Transjugular liver biopsy showed markedly cholestatic hepatitis (modified histology activity index [HAI] inflammatory grade $9 / 18$ and fibrosis 


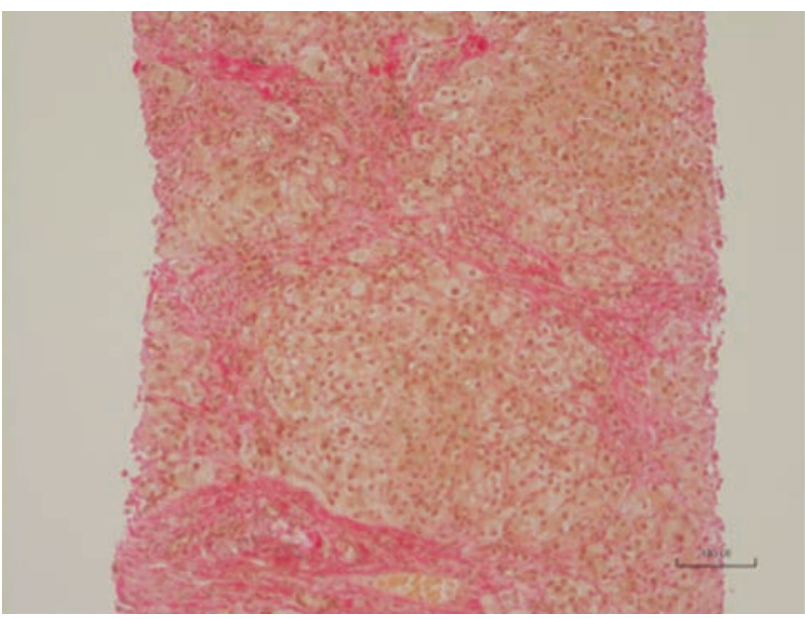

Fig 1. Liver biopsy showing markedly cholestatic hepatitis (modified histology activity index [HAI] inflammatory grade $9 / 18$ and fibrosis stage $5 / 6$ ) in keeping with fibrosing cholestatic hepatitis $B$.

stage 5/6), in keeping with fibrosing cholestatic hepatitis B (Fig 1). He was started on lamivudine in addition to tenofovir, but his clinical condition deteriorated (bilirubin $366 \mu \mathrm{mol} / \mathrm{l}$, albumin $31 \mathrm{~g} / \mathrm{l})$. He required prolonged hospital admission (3 weeks) complicated by poor nutrition and pruritis. Fig 2 shows a graph of his blood tests during the HBV reactivation. His LFTs gradually improved and normalised after 5 months. He is now clinically well and remains on tenofovir.

\section{Key points}

> Patients with current or past hepatitis B (HBV) infection are at risk of viral reactivation if they receive immunemodulating treatment or chemotherapy

> Reactivation can range from subclinical elevation in HBV DNA levels, to severe hepatitis with liver failure and death

> All patients should be screened for hepatitis B with surface antigen and core antibody before receiving immunosuppression

> Patients with positive hepatitis B serology should be referred for specialist advice

> Prophylactic antiviral treatment is recommended for patients with current/past hepatitis $B$ who receive immunosuppressive chemotherapy

\section{Discussion}

The use of rituximab (and other immunosuppressants) is increasing for various autoimmune, haematological and oncological conditions. The purpose of presenting this case is to raise awareness of this potentially life-threatening complication for clinicians who prescribe rituximab and other immunosuppressants. Due to the risk of HBV reactivation with
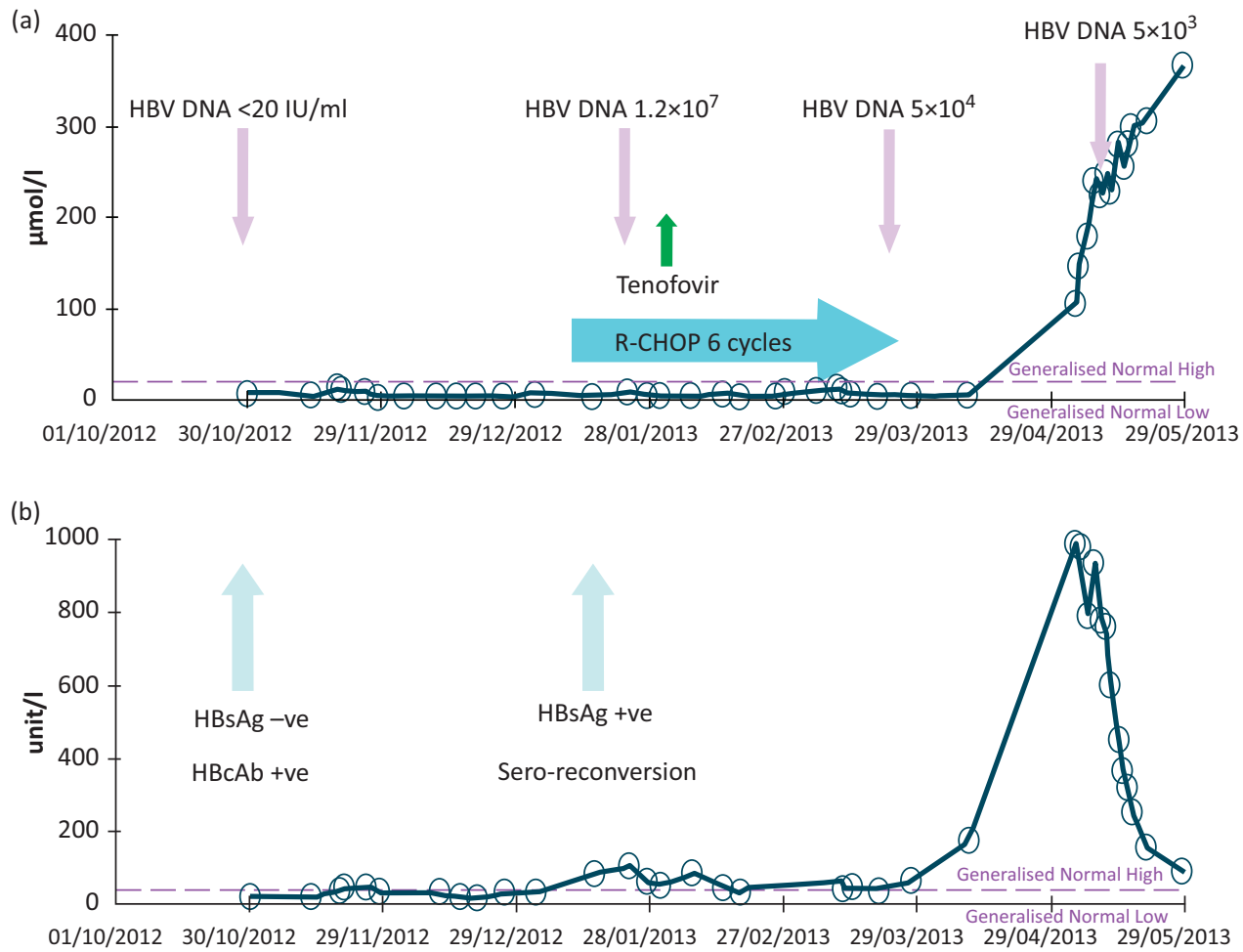

Fig 2. Patient's blood results during the hepatitis $B$ virus (HBV) reactivation. (a) Bilirubin and (b) alanine aminotransferase $(A L T)$. $H B C A b=$ hepatitis $\mathrm{B}$ core antibody; $\mathrm{HBsAg}=$ hepatitis $\mathrm{B}$ surface antigen; $\mathrm{HBV}=$ hepatitis $\mathrm{B}$ virus; $\mathrm{R}-\mathrm{CHOP}=$ rituximab, cyclophosphamide, doxorubicin [hydroxydaunomycin], vincristine, prednisolone. 


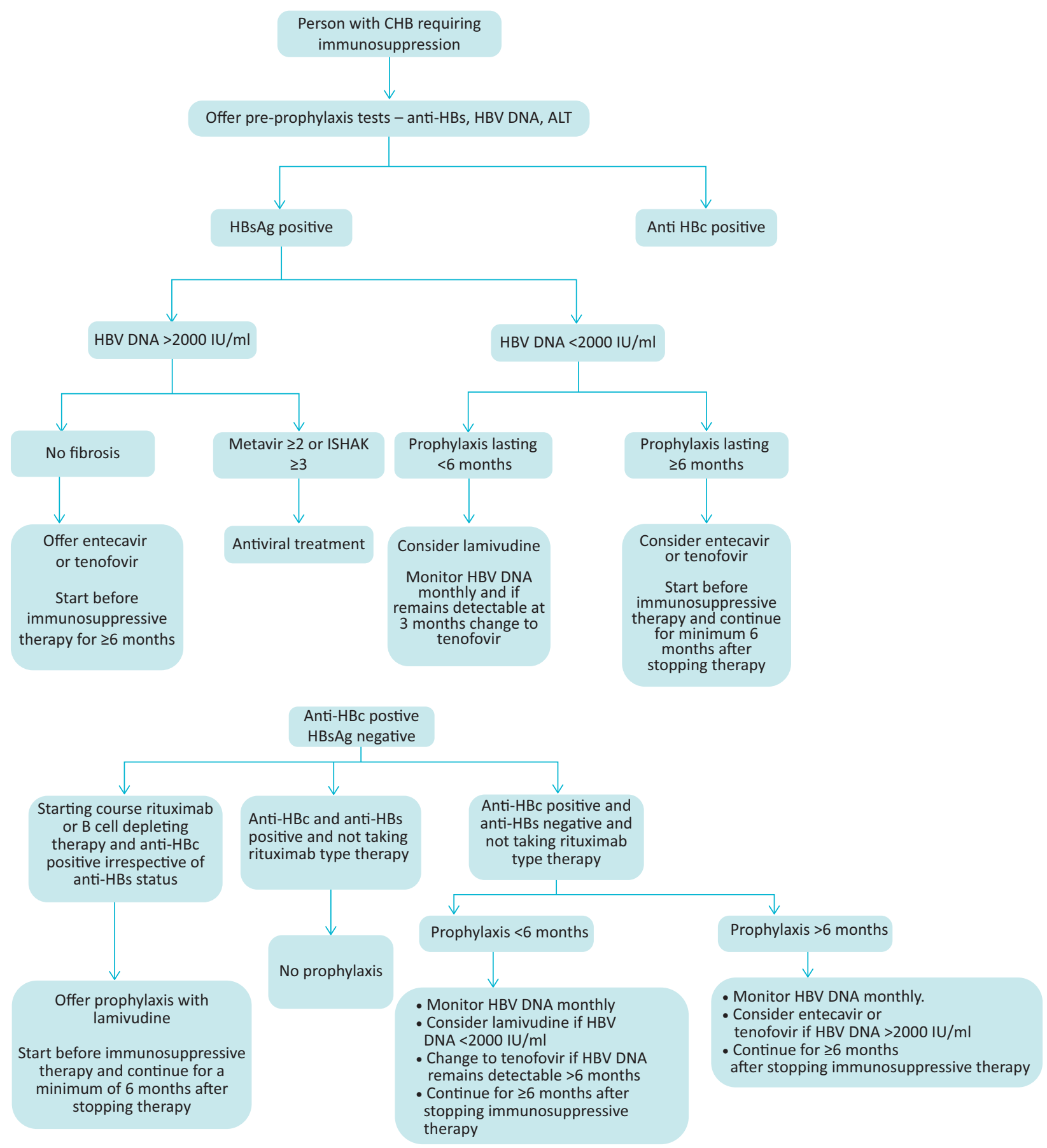

Fig 3. Recommendations of the National Institute for Health and Care Excellence (2013) for the management of hepatitis B in patients receiving immunosuppression and chemotherapy. $\mathrm{ALT}=$ alanine aminotransferase; $\mathrm{CHB}=$ chronic hepatitis $\mathrm{B} ; \mathrm{Anti}-\mathrm{HBC}=$ hepatitis $\mathrm{B}$ core antibody; $\mathrm{HBs} \mathrm{Ag}=$ hepatitis B surface antigen; $\mathrm{HBV}=$ hepatitis B virus; Anti-HBs = hepatitis B surface antibody.

immunosuppression, international guidelines recommend screening all patients for HBsAg and anti-HBcAb before initiating treatment to identify 'at-risk' patients ${ }^{1,2}$ who can be actively monitored or given prophylaxis. Despite these recommendations, HBV screening remains sporadic, with one large US series showing that only $16 \%$ of 18,688 patients were screened before chemotherapy. ${ }^{3}$ Studies assessing HBV screening practices of oncologists suggest that $20-47 \%$ of oncologists do not routinely screen for HBV before treatment, $20 \%$ never screen and others screen 'high-risk' individuals. ${ }^{4,5}$ Zurawska et $\mathrm{al}^{6}$ developed a decision model to compare clinical outcomes, costs and cost-effectiveness of three HBV screening strategies for patients receiving R-CHOP. Screening all patients was the least costly and associated with the highest 1-year 
survival rate (compared with screening 'high risk' and 'none'). Worldwide, chronic HBV infection is very common ${ }^{7}$ and importantly a high proportion of patients with current or past $\mathrm{HBV}$ infection are unaware of the infection, ${ }^{8}$ so it is important to screen all patients who receive immunosuppression/ chemotherapy. ${ }^{9}$

In this case, HBV screening was undertaken before starting $\mathrm{R}-\mathrm{CHOP}$ and the patient was anti-HbcAb positive, but HBsAg negative, in keeping with past infection. The decision was made to monitor for reactivation with LFTs, HBsAg and HBV DNA testing during chemotherapy, as per the guidelines of the European Association of the Study of the Liver (EASL). After 3 months, blood tests showed HBsAg positivity and high HBV DNA, suggesting active HBV. Patients who have had past $\mathrm{HBV}$ infection can reactivate because covalently closed, circular HBV DNA (cccDNA) is archived in hepatocyte nuclei and remains under immune control, ${ }^{10}$ although in states of immunosuppression viral replication can occur, leading to production of HBV DNA and HBsAg and subsequent $\mathrm{HBV}$ reactivation.

When this patient showed serological evidence of HBV reactivation he was treated with tenofovir to suppress viral replication, but 2 months later, after chemotherapy was complete, he developed a very severe fibrosing cholestatic hepatitis. Importantly, the consequences of $\mathrm{HBV}$ reactivation (severe hepatitis) frequently occur after completion of chemotherapy when immune reconstitution occurs and the cytotoxic T-cell-mediated response is restored. ${ }^{11} \mathrm{HBV}$ reactivation can occur anywhere from 2 weeks to more than 1 year after cessation of immunosuppression. ${ }^{9}$ Therefore, ongoing monitoring of patients is essential after chemotherapy, even if they are started on antiviral therapy.

There are many immunosuppressive and chemotherapeutic agents and the absolute risk of HBV reactivation varies with specific regimens, ${ }^{9,11}$ although few studies have assessed risks with specific agents. Overall, studies suggest a reactivation rate of approximately $50 \%$ for HBsAg-positive patients receiving chemotherapy for lymphoma and 15\% for solid tumours. ${ }^{12-16}$ Patients with past infection (anti-HBcAb positive only) have lower reactivation rates (approximately 5\%). ${ }^{17,18}$ More intensive immunosuppressive regimens, particularly those including rituximab and/or high-dose steroids, are the highest risk. ${ }^{9,11}$ Recent studies suggest that reactivation rates in anti-HBcAb-positive patients treated with rituximab are $25 \% .^{19,20}$ The clinical significance of HBV reactivation can be variable, ranging from a mild transaminitis to liver failure and death. Severe reactivation resulting in jaundice occurs in $10-22 \%$ of patients and has a high mortality rate of $4-41 \% .^{12,13,15,21,22}$ As well as being a life-threatening complication, the medical resource costs of $\mathrm{HBV}$ reactivation can be significant, as in this case.

Importantly, HBV reactivation in $\mathrm{HBsAg}$ - or anti-HBcAb-positive patients can be prevented by prophylactic treatment with oral antiviral drugs. ${ }^{23,24}$ Studies have shown that prophylactic antiviral therapy before starting immunosuppression is more effective at preventing hepatitis and reduces mortality compared with initiating treatment once reactivation has occurred. ${ }^{23,24}$ As a result, EASL and the National Institute for Health and Care Excellence (NICE) guidance advises prophylactic antiviral therapy for all HBsAg-positive patients who undergo treatment with immunosuppression or chemotherapy, ${ }^{2}$ and treatment should be continued for at least 6 months after completion of immunosuppressive therapy. ${ }^{25}$ NICE guidelines (Fig 3) recommend treatment with lamivudine if HBV DNA is $<2,000 \mathrm{IU} / \mathrm{ml}$ and the immunosuppression/chemotherapy will last $<6$ months. ${ }^{26}$ For patients whose HBV DNA is $>2,000 \mathrm{IU} / \mathrm{ml}$, or who will receive immunosuppression/ chemotherapy for $>6$ months, entecavir or tenofovir treatment is recommended.

For anti-HBcAb-positive/HBsAg-negative patients receiving immunosuppression/chemotherapy, EASL guidelines recommend ALT and HBV DNA monitoring 1- to 3-monthly and antiviral therapy to be started if the HBV DNA increases. ${ }^{2}$ Our patient was managed according to these recommendations. However, in view of increasing evidence of the high risk of reactivation in anti-HBcAb-positive patients with rituximab, the new NICE guidelines that were published after this patient presented recommend prophylactic treatment with lamivudine. ${ }^{25}$ If our patient had been managed according to these guidelines his reactivation might have been prevented. For anti-HBcAb-positive patients who receive immunosuppressive regimens without rituximab, NICE guidelines recommend monitoring HBV DNA levels monthly and treating with antivirals if HBV DNA increases. ${ }^{25}$

\section{Conclusion}

This case highlights the importance of HBV testing in patients receiving immunosuppression or chemotherapy to identify patients at risk of HBV reactivation, and to treat patients who have current or past HBV infection prophylactically with antiviral therapy to prevent reactivation and the associated morbidity and mortality.

\section{References}

1 Weinbaum CM, Williams I, Mast EE et al. Recommendations for identification and public health management of persons with chronic hepatitis B virus infection. MMWR CDC 2008;57(RR-8): $1-20$.

2 European Association for the Study of the Liver. EASL clinical practice guidelines: Management of chronic hepatitis B virus infection. J Hepatol 2012;57:167-85.

3 Hwang JP, Fisch MJ, Lok AS et al. Trends in hepatitis B virus screening at the onset of chemotherapy in a large US cancer center. BMC Cancer 2013;13:534.

4 Day FL, Link E, Thursky K et al. Current hepatitis B screening practices and clinical experience of reactivation in patients undergoing chemotherapy for solid tumors: a nationwide survey of medical oncologists. J Oncol Pract 2011;7:141-7.

5 Tran TT, Rakoski MO, Martin P et al. Screening for hepatitis B in chemotherapy patients: survey of current oncology practices. Aliment Pharmacol Therapeut 2010;31:240-6.

6 Zurawska U, Hicks LK, Woo G et al. Hepatitis B virus screening before chemotherapy for lymphoma: a cost-effectiveness analysis. J Clin Oncol 2012;30:3167-73.

7 Cooke GS, Main J, Thursz MR. Treatment for hepatitis B. BMJ 2010;340:b5429.

8 McPherson S, Valappil M, Moses SE et al. Targeted case finding for hepatitis B using dry blood spot testing in the British-Chinese and South Asian populations of the North-East of England. J Viral Hepatitis 2013;20:638-44. 
9 Hwang JP, Vierling JM, Zelenetz AD et al. Hepatitis B virus management to prevent reactivation after chemotherapy: a review. Support Care Cancer 2012;20:2999-3008.

10 Rehermann B, Ferrari C, Pasquinelli C et al. The hepatitis B virus persists for decades after patients' recovery from acute viral hepatitis despite active maintenance of a cytotoxic T-lymphocyte response. Nature Med 1996;2:1104-8.

11 Wursthorn K, Wedemeyer H, Manns MP. Managing HBV in patients with impaired immunity. Gut 2010;59:1430-45.

12 Lok AS, Liang RH, Chiu EK et al. Reactivation of hepatitis B virus replication in patients receiving cytotoxic therapy. Report of a prospective study. Gastroenterology 1991;100:182-8.

13 Markovic S, Drozina G, Vovk M et al. Reactivation of hepatitis B but not hepatitis $\mathrm{C}$ in patients with malignant lymphoma and immunosuppressive therapy. A prospective study in 305 patients. Hepato-gastroenterology 1999;46:2925-30.

14 Nakamura Y, Motokura T, Fujita A et al. Severe hepatitis related to chemotherapy in hepatitis B virus carriers with hematologic malignancies. Survey in Japan, 1987-1991. Cancer 1996;78:2210-15.

15 Kumagai K, Takagi T, Nakamura S et al. Hepatitis B virus carriers in the treatment of malignant lymphoma: an epidemiological study in Japan. Ann Oncol 1997;8(Suppl 1):107-9.

16 Yeo W, Chan PK, Zhong S et al. Frequency of hepatitis B virus reactivation in cancer patients undergoing cytotoxic chemotherapy: a prospective study of 626 patients with identification of risk factors. J Med Virol 2000;62:299-307.

17 Uhm JE, Kim K, Lim TK et al. Changes in serologic markers of hepatitis B following autologous hematopoietic stem cell transplantation. Biol Blood Marrow Transplant 2007;13:463-8.

18 Hui CK, Cheung WW, Zhang HY et al. Kinetics and risk of de novo hepatitis B infection in HBsAg-negative patients undergoing cytotoxic chemotherapy. Gastroenterology 2006;131:59-68.
19 Yeo W, Chan TC, Leung NW et al. Hepatitis B virus reactivation in lymphoma patients with prior resolved hepatitis B undergoing anticancer therapy with or without rituximab. J Clin Oncol 2009;27:605-11.

20 Huang YH, Hsiao LT, Hong YC et al. Randomised controlled trial of entecavir prophylaxis for rituximab-associated hepatitis B virus reactivation in patients with lymphoma and resolved hepatitis B. J Clin Oncol 2013;31:2765-72.

21 Liang R. How I treat and monitor viral hepatitis B infection in patients receiving intensive immunosuppressive therapies or undergoing hematopoietic stem cell transplantation. Blood 2009;113:3147-53.

22 Hoofnagle JH, Dusheiko GM, Schafer DF et al. Reactivation of chronic hepatitis B virus infection by cancer chemotherapy. Ann Intern Med 1982;96:447-9.

23 Hsu C, Hsiung CA, Su IJ et al. A revisit of prophylactic lamivudine for chemotherapy-associated hepatitis B reactivation in non-Hodgkin's lymphoma: a randomised trial. Hepatology 2008;47:844-53.

24 Loomba R, Rowley A, Wesley R et al. Systematic review: the effect of preventive lamivudine on hepatitis $\mathrm{B}$ reactivation during chemotherapy. Ann Intern Med 2008;148:519-28.

25 Sarri G, Westby M, Bermingham S et al. Diagnosis and management of chronic hepatitis B in children, young people, and adults: summary of NICE guidance. BMJ 2013;346:\{3893.

26 National Institute for Health and Care Excellence. Hepatitis B (chronic): Diagnosis and management of chronic hepatitis B in children, young people and adults. CG165. London: NICE, 2013. www.nice.org.uk/guidance/cg165 [Accessed 4 August 2014]

Address for corresponding author: Dr JK Dyson, Liver Unit, Freeman Hospital, Newcastle upon Tyne Hospitals NHS Foundation Trust, High Heaton, Newcastle upon Tyne NE7 7DN. Email: jessicadyson@doctors.org.uk 